\title{
FATORES DE RISCO PARA A COLUNA: AVALIAÇÃO EM CONSULTA DE ENFERMAGEM. ${ }^{1}$
}

\author{
SPINE RISK FACTORS: ASSESSMENT AND IMPLEMENTATION OF THERAPEUTIC \\ MEASURES.
}

\author{
Maria Edla de Oliveira Bringuente ${ }^{2}$ \\ Ivana Silveira de Castro ${ }^{3}$ \\ João Carlos Gomes de Jesus ${ }^{4}$ \\ Luzimar dos Santos Luciano ${ }^{3}$
}

\begin{abstract}
RESUMO: O presente trabalho teve como propósito estudar os fatores de risco que afetam a pessoa com dor na coluna, identificando-os e implementando uma proposta de intervenção, composta de um programa de educação à saúde, fundamentado no ensino do autocuidado, pressupostos filosóficos humanistaexistenciais e abordagens equalizadoras do estresse, dentre elas, atividades reintegradoras músculo-esqueléticas, técnicas básicas de equalização do estresse e massagens. Foi desenvolvido junto a uma população de 42 clientes, tendo sido utilizados na coleta de dados dois instrumentos que integraram o protocolo de consultas de enfermagem. Os resultados demonstraram a existência de fatores de risco associados e modificáveis mediante programas de educação à saúde . O processo avaliativo contribuiu para que se tivesse uma visão das medidas terapêuticas, utilizando nessa abordagem cuidados não convencionais, contribuindo, outrossim, na melhoria da qualidade de vida desses clientes.
\end{abstract}

UNITERMOS: Fatores de risco - Dor na coluna - Intervenção de enfermagem.

\begin{abstract}
The present work aimed at studying risk factor that affect people with backpain, identifying them and implementing an intervention proposal of a health education program based on self-care teaching, existential humanist philosophical projects and stress equalization approach line, skeletal-muscle reintegration activities, basic techniques on stress equalization and massage. It has been developed for a population of 42 (forty-two) clients. Two instruments which integrate nursing consultation protocol have been used in data collection. The results showed the existence of associated risk factors which are changeable according to health education programs. The assessment process has contributed for therapeutic measures focus, using non-conventional care methods for this approach providing an improvement to these clients life quality.
\end{abstract}

KEYWORDS: Risk factors - Back pain - Nursing intervention.

\footnotetext{
${ }^{1}$ Trabalho que contou com apoio do CNPq (Conselho Nacional de Pesquisa e Tecnologia)e PróReitoria de Pesquisa e Pós Graduação da UFES ( Universidade Federal do Espírito Santo)

${ }^{2}$ Enfermeira, Livre-Docente em Enfermagem, Professora do Curso de Enfermagem da UFES

${ }^{3}$ Enfermeiras da Secretaria Estadual e Municipal de Saúde. Bolsistas do CNPq

${ }^{4}$ Médico Ortopedista, Professor da UFES. Bolsista do CNPq
} 


\section{INTRODUÇÃO}

A dor na coluna tem sido um sintoma experimentado por cerca de $80 \%$ da população em algum momento de sua vida ${ }^{15}$. São muitas as patologias que causam dor na coluna, tais como, processos traumáticos, fraturas, hérnias discais, mal-formações congênitas, processos inflamatórios infecciosos e nãoinfecciosos, neoplásicos, metabólicos, dentre eles a osteoporose, os degenerativos e aqueles produzidos por problemas mecânico-posturais ${ }^{8,16,19,27,29}$.

Todavia, a dor crônica relacionada a problemas degenerativos, como artrose, freqüenta $65 \%$ das pessoas adultas e tem constituído um problema de Saúde Pública, sendo a segunda causa de afastamento do trabalhador ao serviço, em sua maioria ocorrendo no período de maior atividade laboral. Isso demanda gastos com benefícios sociais e, sobretudo, desequilibra a sua estrutura familiar com a readaptação a mudanças no estilo de vida, comprometendo assim o seu nivel de qualidade ${ }^{15,19,20,21}$.

Assim, as dores na coluna, lombalgias e cérvico-dorso-lombalgias têm sido consideradas como enfermidades sociais presentes no cotidiano de diversos especialistas da área da saúde. No entanto, vários de seus aspectos etiológicos são desconhecidos, o que tem favorecido uma abordagem diversificada para a compreensão do problema, dentre eles, o enfoque de fatores de risco ${ }^{2,3,8,19}$.

A abordagem epidemiológica de fatores de risco tem sido um enfoque muito utilizado na compreensão dos processos mórbidos, especificamente da coluna e na atenção à saúde ${ }^{5,30}$.

Assim, os fatores relacionados ao problema de dor na coluna ou lombalgia podem estar relacionados a variáveis genéticas, psicossociais, ambientais, econômicas e, sobretudo, ao acesso às políticas sociais, educacionais e de saúde ${ }^{15,19}$.

Convém considerar que as precárias condições de vida e saúde, o uso incorreto da mecânica corporal, a pessoa e as suas relações com o trabalho, o lar e o estilo de vida, o acesso às informações, dentre outros, colocam-se como fatores determinantes do processo de adoecer, que reconduziu a uma abordagem do problema lombálgico, tomando como referência, também, a epidemiologia social $2,3,15,23$.

Nessa perspectiva, a intervenção dos problemas possui dimensões políticoeconômicas e sócio-culturais amplas, principalmente quando atenta-se para o fato de que são poucos os trabalhos que, dentro desta vertente, buscam a leitura desse processo mórbido. No entanto, o processo de adoecer deve ser visto como resultante dessas variáveis ${ }^{5}$.

O tratamento da pessoa com dor na coluna, originária de problemas mecânico-posturais, degenerativos e metabólicos, dentre outros, com base em métodos terapêuticos medicamentosos convencionais, tem-se revelado pouco eficaz. A teoria psiconeurológica da dor, formulada por Melzach e Wall, citada em estudos ${ }^{4,19,20}$, aponta a tensão, ansiedade, depressão e sugestão participando na gênese e no combate à dor, estimulando assim novas formas de abordar esse problema.

Os estudos de Jesus ${ }^{15}$, Knoplich ${ }^{19}$ e Santos ${ }^{32}$ estimularam a abordagem do problema mediante uma visão holística e multidisciplinar, compreendendo o ser 
humano como um ser total, histórico, que interage com seu ambiente (natural e social), capaz de transformar-se e transformar as realidades concretas tanto em nivel individual como coletivo. A abordagem multidisciplinar estimulada por esses trabalhos coloca-se como desafio, estimulando a intervenção mediante o ensino para o autocuidado.

Com isso, entende-se a abordagem de fatores de risco como uma metodologia em que se busca a gênese do processo saúde-doença, colocandose também como estimuladora de necessidade de ações educativas, atuando essas ações na prevenção e tratamento do problema da dor na coluna.

Para tanto, o ensino para o autocuidado toma como base pressupostos teórico-filosóficos, de Freire ${ }^{11}$ e Furter ${ }^{12}$, sobre a educação. Para esses autores, o processo educativo deve atuar nas transposições das "situações limites" em que vive o ser humano.

Com o propósito de estudar o problema, foram elaborados os seguintes objetivos: identificar os fatores de risco que estão relacionados à pessoa com o problema de dor na coluna; identificar os fatores de risco que seriam modificáveis mediante programa e plano de intervenção; avaliar, junto à população usuária, as medidas terapêuticas implementadas.

\section{ASPECTOS METODOLÓGICOS}

O estudo é de natureza descritiva com abordagem quanti-qualitativa. Desenvolvido junto ao Serviço de Lombalgia, Ambulatório de Ortopedia do Hospital Universitário Cassiano Antonio de Morais (HUCAM), no período de agosto de 1993 a julho de 1995. A amostra foi constituida de 42 (quarenta e duas) pessoas de um total de 73 (setenta e três) que freqüentaram o programa nesse periodo. O critério de seleção da amostra deu-se em função do aceite e interesse em participar do estudo, tempo médio de permanência no programa e disponibilidade de comparecer às Consultas de Enfermagem (CE). Participaram aqueles que compareceram a mais de três consultas, suficientes para coleta de dados relativos à identificação dos fatores de risco( FR).

O instrumento de coleta de dados, constituído por um Formulário (Anexo 1), foi integrado ao Protocolo de Consulta de enfermagem (PCE), composto de: ficha de consulta de enfermagem à pessoa com espondiloalgia; ficha de avaliação espondiloálgica; plano de intervenção; ficha de acompanhamento e avaliação de aprendizagem e instrumento de avaliação dos cuidados à saúde.

Os dados foram coletados mediante entrevista e avaliação física durante a CE, quando era instituida a estratégia de atendimento constituído por: fase de diagnóstico, objetivos e metas, e plano de intervenção individualizado em que era avaliado e implementado o ensino para o autocuidado a cada retorno do cliente, tendo-o como elemento ativo do processo ensino-aprendizagem.

Após a nona ou décima consulta, o cliente era avaliado quanto ao seu nível de prontidão para o autocuidado, e orientado o seu retorno para controle dentro de 90 (noventa) dias ou em face a alguma necessidade de cuidados. 
Quando do retorno do paciente à Consulta de enfermagem Controle (CEC), no periodo médio de 90 (noventa) dias, procedia-se à coleta de dados sobre a "Avaliação da Intervenção de Enfermagem" (AIE), tomando como base o instrumento (Anexo II).

Dos sujeitos em estudo, foram identificados fatores de risco em 42 clientes, 35 deles realizaram, em média, 9.5 consultas de enfermagem e 16 deles retornaram à consulta de enfermagem controle, para a avaliação da intervenção de enfermagem.

ANÁLISE DE DADOS Inicialmente foram analisados os dados contidos no Instrumento que identificou os fatores de risco, agrupando-os em tabelas. $\mathrm{Na}$ análise qualitativa, foram utilizadas as falas, menções ou outro dado apreendido durante a CE. Em seguida, analisados os dados referentes às AIE.

\section{PRIMEIRO MOMENTO: APRESENTAÇÃO E ANÁLISE DOS RESULTADOS}

Os dados demográficos (Tabela 1) referem-se ao perfil da clientela constituida por $88 \%$ de mulheres e $12 \%$ de homens. Predomina $01^{\circ}$. Grau (57\%) como nivel de escolaridade; destes, $28.5 \%$ incompleto, desenvolvendo atividades compatíveis com o grau de instrução. Como atividades laboral feminina temos, entre outras, do lar, costureira, serventes.

A faixa etária de maior incidência do problema de dor na coluna(59\%) foi de 30 a 50 anos, colocando-se como interface importante devido ao periodo de prémenopausa, quando já se instalam as alterações hormonais que comprometem o metabolismo do cálcio ${ }^{22,34}$

Quanto às condições físicas, (Tabela 2), 90\% não as apresentou boas, com significativo excesso de peso. Identificados hiperplasia mamária, excesso abdominal e o uso incorreto da mecânica corporal, fatores de risco que afetam, tanto a instabilidade da coluna como, em nivel emocional, a auto-imagem.

Aspectos relacionados à pessoa e ao ambiente de trabalho doméstico e familiar (Tabela 4). Nos dados quantitativos apresentados, foram observadas uma série de contradições, acentuadas, no decorrer das CE.. Uma delas foi a jornada de trabalho; mesmo sendo inferior a 40 horas semanais, foi observado pela população feminina uma sobrecarga determinada pelo afazeres domésticos, bastante enfatizada pelo seu desgaste físico, associado às limitações produzidas pela dor e o fato de necessariamente ter que realizá-los. O mesmo ocorreu com a população predominantemente do lar.

Verificou-se que, mesmo sentindo-se preparadas e ajustadas às atividades laborais, eram as pessoas submetidas à sobrecarga física e privação sensorial, fatores estes que contribuem no processo de inadequação ao trabalho. São aspectos importantes mas não valorizados, pelo fato de dependerem de seus empregos para sobreviverem e não lhes restarem outras opções ${ }^{9}$.

Idênticas contradições foram observadas nas relações conflituosas com os chefes, colegas e no relacionamento familiar, atuando como situações estressoras e que contribuíam na exacerbação do quadro de dor. 
Aspectos sobre o estilo de vida: exercicios físicos, recreação, hábito de sono, indumentária, uso do álcool, fumo e outros (Tabela 5), como variáveis importantes por denunciarem fatores de risco aos problemas físicos e emocionais. Dentre os achados, foi ressaltado que $86 \%$ da população não realizava exercícios físicos de forma regular, e desconheciam quaisquer beneficios relacionados a essa prática. Em seguida, o déficit nas atividades que promovem lazer, visto com preocupação em virtude dos pequenos prazeres saudáveis atuarem como medidas anti-estresse. Autores ${ }^{6,28,31,32,33}$, referem que as dores crônicas e o estresse podem levar a pessoa à perda de interesse para com as atividades sociais sendo responsabilizados por quadro de insatisfação, ansiedade, depressão e outros distúrbios referentes à desarmonia emocional, afetando sobremaneira a sua auto-imagem e estima, dado este corroborado no presente estudo. Em relação ao sono foi observado o uso de medicação ansiolítica e analgésica devido à insônia, ao estresse e à dor.

Quanto aos aspectos relativos ao conhecimento e expectativa sobre o problema de saúde (Tabela 6), foi evidenciada falta de acesso a informações sobre saúde e de conhecimentos relacionados ao seu problema de dor na coluna. Esse dado foi corroborado mediante o processo avaliativo, quando $81 \%$ dos clientes se auto-avaliaram e atribuíram-se um escore com valores abaixo de 5(cinco).Apresentaram conhecimentos sobre os principais estressores, sendo um deles a dor e tinham como forma de enfrentamento o choro, a depressão, o isolamento, o uso de medicação, a oração, dentre outros.

A motivação constituiu importante ponto de partida no processo ensinoaprendizagem para o autocuidado, onde $95 \%$ acharam-se motivados. Esse dado pode ser corroborado observando-se a Tabela 3, que se refere à auto-avaliação do cliente ao entrar no programa, relacionando-se após 9 a 10 CE com o momento de sua alta, quando ele próprio considerava-se apto a se autocuidar.

O trabalho teve como propósito, em primeiro lugar, conhecer os FR presentes na pessoa com dor na coluna. Foram identificados aqueles que poderiam ser modificados e trabalhados mediante um programa de ensino para o autocuidado junto à população em estudo. Observou-se que essa população apresentou um perfil de riscos associados, colaborando com os estudos realizados ${ }^{2,15,16,19}$, podendo, em muitos dos seus aspectos, ser trabalhado.

\section{SEGUNDO MOMENTO: CONCEPÇÃO DOS CLIENTES SOBRE AS INTERVENÇÕES DE ENFERMAGEM}

Esses dados foram coletados mediante entrevista, quando do retorno do cliente ao ambulatório, utilizando-se para tanto o instrumento AIE.

Da população em estudo, 42 clientes, 35 freqüentaram em média $9.5 \mathrm{CE}$, aderiram ao tratamento e apresentaram conhecimentos, atitudes e habilidades para se autocuidarem. Desses, apenas 16 (dezesseis) retornaram ao ambulatório no período previsto de 90 (noventa) dias para a CEC. 
TABELA 1 - DADOS DEMOGRÁFICOS: FAIXA ETÁRIA, COR, ESCOLARIDADE, RELIGIÃO E PROFISSÃO / OCUPAÇÃO

\section{DADOS DEMOGRÁFICOS}

\begin{tabular}{|c|c|c|c|}
\hline \multicolumn{3}{|c|}{ SEXO } \\
\hline \multicolumn{2}{|c|}{ FEMININO } & \multicolumn{2}{|c|}{ MASCULINO } \\
\hline Fi & $\%$ & $\mathrm{Fi}$ & $\%$ \\
\hline
\end{tabular}

\begin{tabular}{|c|c|c|c|c|}
\hline \multicolumn{5}{|l|}{ GRUPO ETÁRIO (em anos) } \\
\hline$<20$ & 1 & 2,4 & - & - \\
\hline $20-30$ & 2 & 4,7 & 1 & 2,4 \\
\hline $30-40$ & 8 & 19,0 & 1 & 2,4 \\
\hline $40-50$ & 17 & 40,0 & 2 & 4,7 \\
\hline $50-60$ & 6 & 14,0 & - & - \\
\hline$>60$ & 3 & 7,0 & 1 & 2,4 \\
\hline \multicolumn{5}{|l|}{ COR } \\
\hline BRANCA & 13 & 31,0 & 4 & 9,5 \\
\hline NEGRA & 7 & 17,0 & - & - \\
\hline AMARELA & - & - & - & - \\
\hline PARDA & 17 & 40,0 & 1 & 2,4 \\
\hline \multicolumn{5}{|l|}{ ESCOLARIDADE } \\
\hline ANALFABETO & 3 & 7,0 & 1 & 2,4 \\
\hline $1^{\circ}$ GRAU INCOMPLETO & 12 & 28,5 & 1 & 2,4 \\
\hline $1^{\circ} \mathrm{GRAU}$ COMPLETO & 12 & 28,5 & - & - \\
\hline $2^{\circ} \mathrm{GRAU}$ INCOMPLETO & 4 & 9,5 & - & - \\
\hline $2^{\circ} \mathrm{GRAU}$ COMPLETO & 3 & 7,0 & 2 & 4,7 \\
\hline SUPERIOR INCOMPLETO & 2 & 2,4 & 1 & 2,4 \\
\hline SUPERIOR COMPLETO & 2 & 4,7 & - & - \\
\hline
\end{tabular}

\section{RELIGIAO}

\begin{tabular}{l|r|r|r|c}
\hline & 29 & 69,0 & 4 & 9,5 \\
\hline CATOLICA & 8 & 19,0 & - & - \\
\hline EVANGÉLICA & - & - & - & - \\
\hline ESPIRITA & - & - & 1 & 2,4 \\
\hline NENHUMA & 15 & 35,7 & - & - \\
\hline OCUPAÇAO/PROFISSÁO & 4 & 9,5 & - & - \\
\hline DO LAR & 3 & 7,0 & 1 & 2,4 \\
\hline COSTUREIRA & 3 & 7,0 & 1 & 2,4 \\
\hline SERVENTE & 3 & 7,0 & - & - \\
\hline ESTUDANTE & 3 & 7,0 & - & - \\
\hline CAIXA DE LOJA & 2 & 4,7 & - & - \\
\hline MERENDEIRA & 1 & 2,4 & - & - \\
\hline TECNICO DE ENFERMAGEM & 1 & 2,4 & - & - \\
\hline ADMINISTRADOR & 1 & 2,4 & - & - \\
\hline AJUDANTE DE CAMINHÃO & - & - & 1 & 2,4 \\
\hline BERÇARISTA DE CRECHE & 1 & 2,4 & - & - \\
\hline LAVRADOR & 1 & 2,4 & - & - \\
\hline MECANICO & 1 & 2,4 & - & - \\
\hline PARTEIRO & & & &
\end{tabular}


TABELA 2 - ASPECTOS DAS CONDIÇÕES FISICAS E DE MECÂNICA CORPORAL

\begin{tabular}{|c|c|c|c|c|}
\hline \multirow{3}{*}{ ASPECTOS FÍSICOS } & \multicolumn{4}{|c|}{ AVALIAÇÃO } \\
\hline & \multicolumn{2}{|c|}{ SIM } & \multicolumn{2}{|c|}{ NÃO } \\
\hline & $\mathbf{F i}$ & $\%$ & $\mathbf{F i}$ & $\%$ \\
\hline CONDIÇÕES FÍSICAS SEGUNDO A E* & 04 & 10 & 38 & 90 \\
\hline EXCESSO DE PESO & 25 & 60 & 17 & 40 \\
\hline Leve & 06 & 14 & 19 & 45 \\
\hline Moderado & 04 & 10 & 21 & 50 \\
\hline Alto & 15 & 36 & 10 & 24 \\
\hline HIPERPLASIA MAMÁRIA & 14 & 33 & 28 & 67 \\
\hline Leve & 04 & 10 & 10 & 24 \\
\hline Moderada & 03 & 7 & 11 & 26 \\
\hline Alta & 07 & 17 & 07 & 17 \\
\hline EXCESSO ABDOMINAL & 24 & 57 & 18 & 43 \\
\hline DEFORMIDADE FISICA & 05 & 12 & 37 & 88 \\
\hline Congênita & 01 & 2 & 41 & 98 \\
\hline Adquirida & 04 & 10 & 38 & 90 \\
\hline \multicolumn{5}{|l|}{ CONDIÇÕES DA MECÂNICA CORPORAL: } \\
\hline \multicolumn{5}{|l|}{ Utilizou a mecânica corporal corretamente quando em } \\
\hline pé, sentado ou agachado? & 04 & 10 & 38 & 90 \\
\hline \multicolumn{5}{|l|}{ Distribui o peso corretamente quando: levanta do solo, } \\
\hline transporta e locomove-se? & 11 & 26 & 31 & 74 \\
\hline
\end{tabular}

* Avaliação espondilo-álgica 
TABELA 3 - AVALIAÇÃO DO PROCESSO ENSINO-APRENDIZAGEM PARA O AUTOCUIDADO SEGUNDO A CONCEPÇÃO DOS CLIENTES.

\begin{tabular}{c|c|c|c|c}
\hline \multirow{2}{*}{ ESCORE } & \multicolumn{2}{|c|}{ Entrada no Programa } & \multicolumn{2}{c}{ Saida de Programa } \\
\cline { 2 - 5 } & $\mathbf{F i}$ & $\%$ & $\mathbf{F i}$ & $\%$ \\
\hline 1 & 06 & 14 & 01 & 3 \\
\hline 2 & 12 & 29 & 03 & 8 \\
\hline 3 & 12 & 29 & 02 & 6 \\
\hline 4 & 04 & 9 & 05 & 3 \\
\hline 5 & 02 & 5 & 01 & 6 \\
\hline 7 & 03 & 7 & 02 & 23 \\
\hline 8 & 01 & 2 & 08 & 28 \\
\hline 9 & 02 & 5 & 10 & 6 \\
\hline 10 & - & - & 35 & 100 \\
\hline TOTAL & 42 & 100 & & 3 \\
\hline
\end{tabular}


Fatores de Risio para a Colana : Aôaliaşão ém...

TABELA 4 - ASPECTOS RELACIONADOS À PESSOA E AO AMBIENTE: DE TRABALHO, DOMÉSTICO E FAMILIAR

\begin{tabular}{|c|c|c|c|c|c|c|c|}
\hline \multirow{2}{*}{\multicolumn{2}{|c|}{ ASPECTOS }} & \multicolumn{2}{|c|}{ SIM } & \multicolumn{2}{|c|}{ NÃO } & \multicolumn{2}{|c|}{ EM PARTE } \\
\hline & & $\mathbf{F i}$ & $\%$ & $\mathbf{F i}$ & $\%$ & $\mathbf{F i}$ & $\%$ \\
\hline \multirow[t]{2}{*}{ JORNADA DE TRABALHO: } & \multirow{2}{*}{$\begin{array}{l}<40 \text { horas }(23)^{*} \\
>40 \text { horas }\end{array}$} & 21 & 91 & 02 & 9 & - & - \\
\hline & & 02 & 9 & 21 & 91 & - & - \\
\hline \multicolumn{2}{|c|}{ PREPARO PARA 0 TRABALHO : $(23)^{*}$} & 16 & 70 & 07 & 30 & - & - \\
\hline \multicolumn{2}{|c|}{ FREQUÊNCIA DA SOBRECARGA FISICA: $(23)^{*}$} & 15 & 65 & 08 & 35 & - & - \\
\hline \multirow{3}{*}{\multicolumn{2}{|c|}{$\begin{array}{ll}\text { regular } \\
\text { eventual } \\
\text { periódica } \\
\end{array}$}} & 13 & 57 & - & - & - & - \\
\hline & & 02 & 9 & - & - & - & - \\
\hline & & - & - & - & - & - & - \\
\hline \multirow{4}{*}{\multicolumn{2}{|c|}{$\begin{aligned} \text { CARGA A QUE SE SUBMETE : } & \\
& \text { leve } \\
& \text { moderada } \\
& \text { alta }\end{aligned}$}} & & & & & & \\
\hline & & 02 & 9 & - & - & - & - \\
\hline & & 09 & 39 & - & - & - & - \\
\hline & & 07 & 30 & - & - & - & - \\
\hline \multirow{6}{*}{\multicolumn{2}{|c|}{$\begin{array}{l}\text { POSIÇÃO CORPORAL DURANTE O TRABALHO } \\
\text { Andando }(23)^{*} \\
\text { Em pé } \\
\text { Sentado } \\
\text { De cócoras } \\
\text { Deitado } \\
\text { Outros }\end{array}$}} & 06 & 26 & - & - & - & - \\
\hline & & 07 & 30 & - & - & - & - \\
\hline & & 07 & 30 & - & - & - & - \\
\hline & & - & - & - & - & - & - \\
\hline & & - & - & - & - & - & - \\
\hline & & 03 & 14 & - & - & - & - \\
\hline \multicolumn{2}{|c|}{ TIPOS DE PRIVAÇÃO SENSORIAL: $(23)^{*}$} & 17 & 74 & 06 & 26 & - & - \\
\hline \multirow{5}{*}{\multicolumn{2}{|c|}{$\begin{array}{ll}\text { Ruídos sonoros } \\
\text { Temperatura } \\
\text { Umidade } \\
\text { Visual } \\
\text { Outras: inalação de gases }\end{array}$}} & 12 & 52 & - & - & - & - \\
\hline & & 07 & 30 & - & - & - & - \\
\hline & & 01 & 4 & - & - & - & - \\
\hline & & 02 & 9 & - & - & - & - \\
\hline & & 01 & 4 & - & - & - & - \\
\hline \multirow{5}{*}{\multicolumn{2}{|c|}{$\begin{array}{l}\text { EMOCIONAIS NO TRABALHO: }(23)^{*} \\
\qquad \begin{aligned} \text { Sente-se ajustado } \\
\text { Gosta do que faz } \\
\text { Gosta do ambiente de trabalho } \\
\text { Sente-se estimulado p/o trabalho que realiza }\end{aligned}\end{array}$}} & & & & & & \\
\hline & & 16 & 70 & 03 & 13 & 04 & 17 \\
\hline & & 17 & 74 & 02 & 9 & 04 & 17 \\
\hline & & 17 & 74 & 02 & 9 & 04 & 17 \\
\hline & & 15 & 65 & 03 & 13 & 05 & 22 \\
\hline \multicolumn{8}{|c|}{ RELACIONA-SE BEM COM : } \\
\hline \multirow{2}{*}{\multicolumn{2}{|c|}{$\begin{array}{l}\text { Chefe }(18)^{*} \\
\text { Colegas }\end{array}$}} & 12 & 67 & 03 & 14 & 03 & 14 \\
\hline & & 16 & 89 & 01 & 60 & 01 & 60 \\
\hline \multicolumn{8}{|c|}{ AMBIENTE DOMÉSTICO: $(42)^{*}$} \\
\hline \multirow{2}{*}{\multicolumn{2}{|c|}{$\begin{array}{l}\text { Físico } \\
\text { Emocional }\end{array}$}} & 32 & 76 & 04 & 10 & 06 & 14 \\
\hline & & 22 & 52 & 05 & 12 & 15 & 36 \\
\hline \multicolumn{2}{|c|}{$\begin{array}{l}\text { DESENVOLVIMENTO DAS ATIVIDADES DOMÉSTICAS }(42)^{*} \text { : } \\
\text { Gosta de realizar atividades domésticas }\end{array}$} & 16 & 38 & 16 & 38 & 10 & 24 \\
\hline \multirow{6}{*}{\multicolumn{2}{|c|}{$\begin{array}{l}\text { RELACIONAMENTO HAMONIOSO COM FAMILIARES: } \\
\qquad \begin{aligned} \text { Familia }(42)^{*} \\
\text { Parceiro }(42)^{*} \\
\text { Filhos }(39)^{*} \\
\text { Pai }(36)^{*} \\
\text { Mãe }(27)^{*} \\
\text { Irmão }(42)^{*}\end{aligned}\end{array}$}} & 23 & 55 & 05 & 12 & 14 & 33 \\
\hline & & 21 & 54 & 04 & 10 & 14 & 36 \\
\hline & & 32 & 89 & 01 & 4 & 03 & 8 \\
\hline & & 22 & 81 & 01 & 4 & 04 & 15 \\
\hline & & 22 & 81 & 03 & 11 & 02 & 7 \\
\hline & & 30 & 71 & 05 & 12 & 07 & 17 \\
\hline
\end{tabular}

( )* Considerando como base para cálculo para cada variável em estudo. 
TABELA 5 - ASPECTOS SOBRE O ESTILO DE VIDA : EXERCÍCIOS FISICOS, RECREAÇÃO, HÁBITOS DE SONO E INDUMENTÁRIA, USO DE ÁLCOOL, FUMO E OUTROS $(42=100 \%)^{*}$.

\begin{tabular}{|c|c|c|c|c|c|c|}
\hline \multirow[b]{2}{*}{ ASPECTOS / ESTILOS DE VIDA } & \multicolumn{2}{|c|}{ SIM } & \multicolumn{2}{|c|}{ NÃO } & \multicolumn{2}{|c|}{ EM PARTE } \\
\hline & $\mathrm{Fi}$ & $\%$ & $\mathrm{Fi}$ & $\%$ & $\mathrm{Fi}$ & $\%$ \\
\hline TIPOS DE EXERCICIOS FÍSICOS & 06 & 14 & 36 & 86 & - & - \\
\hline \multirow{2}{*}{$\begin{array}{l}\text { Coletivo } \\
\text { Individual }\end{array}$} & 04 & 10 & -- & -- & - & - \\
\hline & 02 & 05 & -- & - & - & - \\
\hline ATIVIDADES DE LAZER & 28 & 67 & 14 & 33 & - & - \\
\hline \multirow{3}{*}{$\begin{array}{l}\text { Regular } \\
\text { Eventual } \\
\text { Periódica } \\
\end{array}$} & 03 & 07 & -- & -- & - & - \\
\hline & 13 & 31 & -- & -- & - & - \\
\hline & 12 & 29 & -- & -- & - & - \\
\hline \multicolumn{7}{|l|}{ HÁBITOS DE SONO: } \\
\hline & 28 & 66 & 10 & 24 & 4 & 10 \\
\hline \multirow{2}{*}{$\begin{aligned} \text { Número de horas dormidas: } & <04 \text { horas } \\
& >04 \text { horas }\end{aligned}$} & 7 & 17 & -- & -- & - & - \\
\hline & 35 & 83 & -- & -- & - & - \\
\hline \multicolumn{7}{|l|}{ POSIÇÃO QUE ASSUME QUANDO DORME : } \\
\hline \multirow{3}{*}{$\begin{array}{c}\text { Ventral } \\
\text { Lateral } \\
\text { Dorsal } \\
\text { Outros: Posição Fetal }\end{array}$} & 29 & 70 & -- & -- & - & - \\
\hline & 1 & 2 & -- & -- & - & - \\
\hline & 1 & 2 & -- & -- & - & - \\
\hline USO DE COLCHÃO ANATÔMICO. & 31 & 74 & 11 & 26 & - & - \\
\hline \multirow{3}{*}{$\begin{array}{l}\text { FAZ USO DO TRAVESSEIRO } \\
\text { Altura do travesseiro : até } 10 \mathrm{~cm} \\
>10 \mathrm{~cm}\end{array}$} & 40 & 95 & 2 & 5 & - & - \\
\hline & 22 & 55 & 18 & 45 & - & - \\
\hline & 18 & 45 & 22 & 55 & - & - \\
\hline \multicolumn{7}{|l|}{ USO DE INDUMENTÁRIA } \\
\hline \multirow{3}{*}{$\begin{aligned} & \text { Sapato Apertado } \\
& \text { Salto do sapato: Altura }<5 \mathrm{~cm} \\
&>5 \mathrm{~cm}\end{aligned}$} & 0 & 0 & 42 & 100 & - & - \\
\hline & 34 & 81 & 8 & 19 & - & - \\
\hline & 8 & 19 & 34 & 81 & - & - \\
\hline \multirow{2}{*}{$\begin{array}{l}\text { HÁBITOS ALIMENTARES } \\
\text { FAZ USO DE ALIMENTAÇÃO BALANCEADA }\end{array}$} & & & & & & \\
\hline & 12 & 29 & 9 & 21 & 21 & 50 \\
\hline \multirow{5}{*}{$\begin{array}{l}\text { Proteínas } \\
\text { Carboidratos } \\
\text { Lipídeos } \\
\text { Vitaminas } \\
\text { Sais minerais }\end{array}$} & 21 & 50 & 21 & 50 & - & - \\
\hline & 24 & 57 & 18 & 43 & - & - \\
\hline & 21 & 50 & 21 & 50 & - & - \\
\hline & 11 & 26 & 31 & 74 & - & - \\
\hline & 8 & 19 & 34 & 81 & - & - \\
\hline OUTROS: EXPOSIÇÃO AO SOL & 35 & 83 & 7 & 17 & - & - \\
\hline \multirow{3}{*}{$\begin{array}{ll}\text { Regular } \\
\text { Eventual } \\
\text { Periódico }\end{array}$} & 11 & 26 & -- & -- & - & - \\
\hline & 15 & 36 & -- & -- & - & - \\
\hline & 9 & 21 & - & -- & - & - \\
\hline \multirow{3}{*}{$\begin{array}{l}\text { FAZ USO DO FUMO } \\
\qquad \begin{aligned} \text { Quantidade: } & <10 \text { cigarros/dia } \\
& >10 \text { cigarros/dia }\end{aligned}\end{array}$} & 9 & 21 & 33 & 79 & - & - \\
\hline & 3 & 7 & -- & -- & - & - \\
\hline & 5 & 12 & -- & -- & - & - \\
\hline \multirow{4}{*}{$\begin{array}{r}\text { FAZ USO DO ÁLCOOL } \\
\qquad \begin{array}{r}\text { Regular } \\
\text { Eventual } \\
\text { Periódico }\end{array} \\
\end{array}$} & 10 & 24 & 32 & 76 & - & - \\
\hline & 0 & 0 & - & -- & - & - \\
\hline & 5 & 12 & -- & -- & - & - \\
\hline & 5 & 12 & - & - & - & - \\
\hline
\end{tabular}

( * ) Considerado como base no cálculo para cada variável em estudo. 


\section{TABELA 6 - ASPECTOS RELACIONADOS AO CONHECIMENTO E EXPECTATIVA SOBRE O PROBLEMA DE SAÚDE (42=100\%).}

\begin{tabular}{|c|c|c|c|c|c|c|}
\hline \multirow[b]{2}{*}{ ASPECTOS/CONHECIMENTO E EXPECTATIVA } & \multicolumn{2}{|c|}{ SIM } & \multicolumn{2}{|c|}{ NÃO } & \multicolumn{2}{|c|}{ EM PARTE } \\
\hline & Fi & $\%$ & $\mathbf{F i}$ & $\%$ & $\mathbf{F i}$ & $\%$ \\
\hline TEM ACESSO A INFORMAÇÕES SOBRE SAÚDE: * & 36 & 86 & & & & \\
\hline Televisão & 23 & 58 & 06 & 14 & - & - \\
\hline Profissionais de Saúde & 15 & 38 & - & - & - & - \\
\hline Livros & 03 & 7 & - & - & - & - \\
\hline Revista & 02 & 5 & - & - & - & - \\
\hline Jornais & 02 & 5 & - & - & - & - \\
\hline Rádio & 02 & 5 & - & - & - & - \\
\hline POSSUI CONHECIMENTO FUNDAMENTADO SOBRE A DOENÇA: & 11 & 26 & 31 & 74 & - & - \\
\hline \multicolumn{7}{|l|}{ POSSUI CONHECIMENTO SOBRE O MECANISMO: } \\
\hline da dor & 06 & 14 & - & - & - & - \\
\hline da atrofia muscular & 03 & 7 & - & - & - & - \\
\hline da parestesia & 02 & 5 & - & - & - & - \\
\hline \multirow{2}{*}{\multicolumn{7}{|c|}{$\begin{array}{l}\text { CONHECIMENTO SOBRE O PROBLEMA } \\
\text { ESCORE DE } 1 \text { A } 10 \text { ATRIBUÍDO PELO CLIENTE: }\end{array}$}} \\
\hline & & & & & & \\
\hline$<5$ & 34 & 81 & - & - & - & - \\
\hline$>5$ & 08 & 19 & - & - & - & - \\
\hline \multicolumn{7}{|l|}{ ESCORE DE 1 A 10 ATRIBUÍDO PELO ENFERMEIRO: } \\
\hline$<5$ & 34 & 81 & - & - & - & - \\
\hline$>5$ & 8 & 19 & - & - & - & - \\
\hline POSSUI O CONHECIMENTO DAS PISTAS ESTRESSORAS: & 33 & 79 & 09 & 21 & - & - \\
\hline DESENVOLVE HABILIDADES COGNITIVAS NO CONTROLE DO ESTRESSE: & 29 & 69 & 13 & 31 & - & - \\
\hline ENCONTRA-SE MOTIVADO PARA APRENDIZAGEM & 40 & 95 & 02 & 5 & - & - \\
\hline NUTRE ESPERANÇAS DIANTE DA VIDA: & 32 & 76 & 10 & 24 & - & - \\
\hline POSSUI PADRÃO POSITIVO DE PENSAMENTO & & & & & & \\
\hline Em Relação ao Problema & 22 & 52 & 12 & 25 & 8 & 19 \\
\hline Em Relação Auto-Imagem & 21 & 50 & 13 & 31 & 8 & 19 \\
\hline
\end{tabular}

*Fonte de Informações: não houve excludência, foi trabalhada a freqüência na ordem de apresentação. 
A concepção dos clientes sobre o plano de intervenção, fundamentado no ensino do autocuidado e medidas equalizadoras do estresse, pode ser compreendida mediante uma abordagem subjetiva e objetiva: as falas, o fácies comportamento apresentado quando do retorno à CE, dentre outros.

Observa-se que as atividades desenvolvidas no programa eram realizadas pelos clientes de forma regular e que as mesmas contribuiam para o "alívio da dor, diminuição da tensão e como forma de enfrentar e melhorar o estado de dor". As mais citadas: Atividades reintegradoras músculo-esqueléticas (ARME);Técnicas básicas de equalização do estresse (TBEs), Massagem e auto-massagem (MA), seguidas de Caminhadas; Espacialidade corporal (EC); Calor local ,Sol e Dança. Essas medidas terapêuticas foram fundamentadas em autores que buscam a compreensão da pessoa como ser total,

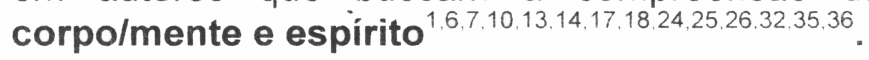

É importante observar que todos os hábitos mencionados, como: "cuidado com a postura; aprender a pegar peso de forma correta; correção da posição de dormir" dentre outras, e que sofreram mudanças foram colocados como fatores de risco modificáveis, com o uso de cuidados tão simples, mas de fundamental importância à saúde e, especificamente, da pessoa com problema de coluna.

A importância do ensino para o autocuidado no controle e enfrentamento da dor e do estresse pode ser compreendida através de suas falas durante a avaliação das intervenções:

As atividades reintegradoras músculo-esqueléticas (ARME): Ajudaram no alizio da dor e melhora dos movimentos do meu corpo; É bom, quando começo a sentir tensão nas costas faço e logo melhoro.

A massagem nas costas e nos pés realizadas durante as CE: Pára a dor, relaxa e dá uma sensação boa; As mãos, o calor melhora muito a dor; Solta os nervos. Fiquéi mellior dos pés dormentes;

A automassagem, com bola de gude e o rolo de cabo de vassoura: Ajudou a melhorar a dor do calcanhar; Amolece os nervos e descansa os pés;

As técnicas básicas de equalização do estresse : Faço todos os dias, melhora o meu nervoso. ;

Atividade mobilizadora para o autocuidado: Achei bom porque aprendi a tratar de minha coluna. Foi bom saber do estresse e eu que sou uma pilla;

Espacialidade corporal: Foi bom conversar com o nosso corpo; foi bom ver as juntas se moverem;

A eletromagnetoterapia: Ótimo. Chegaza tensa, usava o aparelho e quase que sumia a dor nas costas;

A dança: Ajuda a fazer os exercícios ao som da música, eu relaxo. Mexe com todas as juntrs. 
Quanto à frequência e intensidade em relação ao episódio da dor, observase, após o ensino de ações para o auto-cuidado, que houve melhoras, levando à sua diminuição . O tempo não foi uma questão bem delimitada .Todavia, o que pode ser inferido é que as pessoas passaram a se compreender melhor, tiveram acesso ao conhecimento sobre o fenômeno da dor na coluna, aprenderam a conhecer-se, cuidar de sua saúde e do seu corpo, trabalharam os seus limites, a sua temporalidade e suas potencialidades.

Isso tudo nos levou a reiterar a crença de que o direito ao acesso à informação sobre a saúde, assim como a oportunidade de as pessoas vivenciarem cuidados com o uso de práticas ou medidas terapêuticas não convencionais, vêm se tornando de fundamental importância. Da mesma forma, o desenvolvimento de trabalhos que estimulem o potencial da pessoa para o auto-cuidado como forma de intervir nos fatores de riscos modificáveis.

\section{CONCLUSÕES}

Foi possível observar na população em estudo a existência de riscos associados, tanto dentro da mesma variável como em seu conjunto, observandose em um mesmo sujeito do estudo a existência de um somatório de eventos indesejáveis atuando no seu desequilibrio físico-mental.

O perfil demográfico nos mostrou a incidência do problema em uma população que diferiu em termos de ocupação daquelas já estudadas em trabalhadores de indústria, da saúde, dentre elas a de enfermagem. No entanto, houve incidência maior em pessoas com baixo nível de escolaridade; de ocupação e atividade laboral que se submetiam à sobrecarga física aliada a uma história de vida que denunciava desgaste físico e eventos desagradáveis. Mesmo assim, tornou-se difícil estabelecer uma correlação direta de dor na coluna com as variáveis em estudo.

Todavia, um dado preocupante reflete o alto índice de mulheres na faixa etária de 40-50 anos com manifestações de problemas na coluna, que se iniciam já na segunda ou terceira década de vida. A osteoporose constitui uma das ameaças a essa população, carecendo de um conjunto de ações preventivas ${ }^{33}$.

A mudança do estilo de vida apresentou-se como variável de risco, com possibilidade de ser trabalhada, mediante o ensino para o autocuidado, desenvolvido de forma dinâmica, criativa, com base em princípios sóciofilosóficos e psico-pedagógicos que considera, dentre outros aspectos, a condição histórica do ser humano, com potencial de desenvolvimento do nascimento à morte ${ }^{11,12}$.

Vimos que os fatores de risco que afetam a pessoa com dor na coluna indicam diversidades e complexidades que se colocam como verdadeiro desafio profissional, necessitando de novos estudos, entre eles, os que envolvem a saúde da criança, para que de forma bastante precoce internalizem-se hábitos de cuidados à saúde. 


\section{REFERÊNCIAS BIBLIOGRÁFICAS}

1. ALEXANDRE, N.M.C; ANGERAMI, E. L. S. Estilo e vida e trabalho do pessoal de Enfermagem e ocorrência de cervicodorsolombalgia. $R$. Lat. Am. de Enferm., Ribeirão Preto, v. 3, n. 1, p. 117-136, jan. 1995.

2. ALVES, J.G., MELO FILHO, J: CORDEIRO, H. Estresse. Jor. Bras. de Medicina, v. 62, n. 4, abr., p.38-42, 1992

3. ATRA, E. Artrose. Revista de Clínica Médica, v. 28, n. 4, p.104-113,mai., 1995.

4. BRANDÃO, M.L. As bases psicofisiológicas do comportamento. São Paulo: EPU, 1991. p. 106-117.

5. BREILH,J. Epidemiologia: Economia, Política e Saúde. 5. ed. Trat. Luís Roberto de Oliveira. São Paulo: Ed. Universidade Estadual Paulista /HUCITEC, 1991. $276 \mathrm{p}$.

6. BRUHNS, H. et al. Conversando sobre o corpo. 3. ed. Campinas. São Paulo: Papirus. 1989. $106 \mathrm{p}$.

7. BRUNNER,L.S.; SUDDARTH, D.S. Tratado de Enfermagem MédicoCirúrgica. 5. ed .Rio de Janeiro: Guanabara, 1987. p.106-117.

8. COUTO, H. A Fisiologia do trabalho aplicado. Belo Horizonte.: Ibéria $1978,295 \mathrm{p}$.

9. DANIELLOU, F.; LAVILLE, A.;TEIGER, C. Ficção e realidade do trabalho operário. R. Bras. de Saúde Ocup., v. 17, n. 66, p. 413, 1989.

10. FAST, J. A linguagem do corpo. Trad. Cristina Rocha. São Paulo: Edições. 70, 1970. 178p.

11. FREIRE, P. Educação como prática de liberdade. 12. ed. São Paulo: Paze Terra, 1981. $150 \mathrm{p}$.

12. FURTER, P. Educação e reflexão. 14 ed. Rio de Janeiro: Paz e Terra, 1984. 190p.

13. IDICULLA, A.;GALDBERG, G. Preparação física para mulheres maduras. In: Clin, Med. da Am. do Norte. Rio de Janeiro: Interlivros, 1987. v.1, p. 125-137. 
14. IWANOWISCZ, B. A imagem e a consciência do corpo. In: Conversando sobre o corpo. 3 ed. Campinas- São Paulo: Papirus. 1989. p 63-81.

15. JESUS, L. Z. Dor Lombar. Um problema mundial. Revista Proteção, Novo Hamburgo, n. 2, p. 18-19. 1988.

16. JESUS, J. C. G. et al. Estudo da perda óssea em pacientes espondiloálgicos do sexo feminino. In: XII SIPUFES, Vitória-ES, Anais ... Vitória-ES: UFES, 1993.

17. KEATING, K. Terapia do braço. Trad. Paulo Rebouças. São Paulo: Ed. Pensamentos, 1983.

18. KISNER ,C. : COLBY, L.A. Exercícios terapêuticos. Fundamentos e técnicas. Trad. Lilian B. Ribeiro. São Paulo: Manole, 1992.

19. KNOPLICH, J. As dores na coluna na medicina do trabalho. R. Bras. de Saúde Ocup., v. 8, n.32, p. 50-52. 1980

20. A importância das dores na coluna na prática médica e industria. ver. R. Bras. de Saúde Ocup., v. 9, n. 16, p. 71- 74. 1981.

21. Lombalgia na indústria. Uma nova opção para tratamento. R. Bras. de Saúde Ocup., v. 10, n. 39. p. 81-84, 1982

22. LANE,E.; PINOTTI, J. A. A mulher e seu climatério. Ginec. e Obstet. Bras. Dep. Gineco. e Obst. USP/ HC, v.10, (3), p. 150-154, 1987.

23. LAVILLE, A. Postural stress in high speed precision work. Ergonomics, 28 (1) , p. 229-236, Jan. 1995

24. LOWEN, A,; LOWEN, L. Bioenergética. Trad. Maria Silva M. Netto. São Paulo: Summus. 1982

25. LUCASINI JÚNIOR, R,; LIMA, V. L. Osteoporose: exercício como prevenção e tratamento. ARS CURANDI, Rev. de Clín. Méd. , v. 27, n. 6, p. 28-36, jul. 1994

26. MEDINA, J. P. S. O brasileiro e seu corpo. Educação e política do corpo. Campinas. São Paulo: Papirus, 1987. 
27. NATOUR, J. Lombalgia. ARS CURANDI- Revista de Clinica Médica, v. 28, n. 25, p.41-48. jun. 1995

28 NUNES, A. M. P. Motivação para o autocuidado um diagnóstico insdispensável na assistência e orientação ao diabético. Texto $e$ Contexto- Enfermagem. Florianópolis. v. 2, n. 1, p. 53-66. jan./jun., 1993.

29. NUNES, C. V. Discopatias cervicais e lombares. Lombociatalgias, Cervicobraquialgias: sintese clinica e tratamento ARS CURANDI- Manual de conduta do clínico geral, v. 20, n. 10, p.68-78 nov./dez., 1987.

30. RIIHIMAKI, H. et al. Low-back, its origin and risk indicators. Scand J. Work. Environ health, v. 17, n. 2, p. 81-90, 1991.

31. RODRIGUES A. L. Estresse e trabalho. PSICODRAMA. Ano 2, n. 2 , p. 7-8, 1988.

32. SANTOS, V. Proposta alternativa de ensino e aprendizagem para o auto-cuidado: uma contribuição da enfermagem ao enfrentamento da dor e equalização do estresse. (Tese de doutorado). USP, E. Enf. Ribeirão Preto, 1990, 152 p.

33. SOUZA, F. P.. Ruído- riscos ao sono. Revista Proteção. v. 5, n. 23, p. 148. jun./jul., 1993.

34. SZENJNFELD, V. L.; BARACAT, E. C. Osteoporose e climatério . ARS CURANDI- Clínica Médica, v. 27, n. 8, p. 61-78, set., 1994

35. The Sunday Telegraph Magnetic beds. The hidden attractions.SUNDAY MATTERS, London, 27 march, 1994.

36. TOMEY,L.; TAYLOR, SPINE, J. Update exercise and spinal manipulation in the tretment of low. Back pain, Spine, v. 20, n. 5, 1995. 